\title{
Totally Implantable Venous Access Device (Chemoport) in Oncology: Study of 168 Polyurethane Chemoport Catheter System
}

\author{
Gyanendra Swaroop Mittal ${ }^{1}$ Deepak Sundriyal2,๑ \\ ${ }^{1}$ Department of Surgery, Santosh Medical College and Hospital, \\ Ghaziabad, Uttar Pradesh, India \\ 2Department of Medical Oncology, Hematology, All India Institute \\ of Medical Sciences, Rishikesh, Uttarakhand, India \\ ${ }^{3}$ Department of Surgical Oncology, Fortis Memorial Research \\ Institute, Gurugram, Haryana, India
}

\section{South Asian J Cancer 2021;10:261-264.}

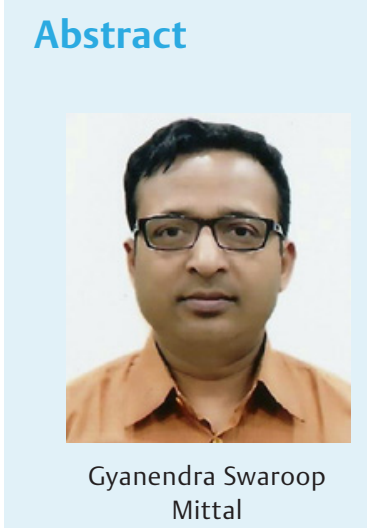

Keywords

- TIVAD

- polyurethane

- chemoport
Background Chemoport (totally implantable venous access device) and its catheter system are used to administer long-term chemotherapy in cancer patients. The objective of this study was to analyze the complications associated with chemoport insertion in various cancer patients.

Material and Methods A total number of 168 chemoports along with polyurethane catheters were inserted in various cancer patients over a period of 3 years. 9.6 F polyurethane catheters were put by a team of surgical oncologists in operation theater under general or local anesthesia. Analysis of the complications was done until the chemoport was removed due to any reason.

Results Out of 168 patients, 30 (17.85\%) developed complications. Complications included arterial puncture, malposition of the catheter tip, pneumothorax, hematoma, seroma, deep vein thrombosis, fracture of the catheter, a reversal of port, infections, and thrombosis of the catheter. Only a few required premature port and catheter removal. Conclusion There was a low rate of complications associated with chemoport using a polyurethane type of catheter system. However, infection-related complications were comparatively more common in our series. Chemoport requires expert handling, patient education, strict follow-up, and dedicated teamwork to minimize complications.

\section{Introduction}

Totally implantable venous access device (TIVAD) or the chemoport was introduced in the year 1982 among patients of cancer for the administration of intravenous (IV) chemotherapy. It was then possible to deliver highly vesicant chemotherapy drugs with minimum morbidity. ${ }^{1}$ Other uses of chemoport are the administration of parenteral nutrition, blood products transfusion, antibiotics and IV fluid administration, and repeated venous sampling, thus avoiding frequent cannulation., ${ }^{2,3}$ Chemoport has a long dwell time and thus prone to complications. Minor complications like hematoma, ecchymosis at the puncture site, and inability to withdraw blood occur frequently but these are of lesser clinical significance as they cause minimal morbidity to the patient. Major complications like venous thrombosis, pneumothorax, infection, fracture of the catheter, or malfunction ture removal of the chemoport.

(C) 2021. MedIntel Services Pvt Ltd.

This is an open access article published by Thieme under the terms of the Creative Commons Attribution-NonDerivative-NonCommercial-License, permitting copying and reproduction so long as the original work is given appropriate credit. Contents may not be used for commercial purposes, or adapted, remixed, transformed or built upon. (https://creativecommons.org/licenses/by-nc-nd/4.0/).

Thieme Medical and Scientific Publishers Private Ltd A-12, Second Floor, Sector -2, NOIDA -201301, India are of major clinical significance, as they may require prema- 
Chemoport insertion is routinely being practiced by surgical oncologists at Dharamshila Hospital and Research Centre for more than 10 years. Scarcity of data on the study of polyurethane catheter systems led to the inception of this study. The objective of this study was to analyze the outcome and complications related to polyurethane catheter system among a variety of cancer patients.

\section{Methods}

This study was performed over a period of 3 years in 168 patients diagnosed with various solid organ and hematological malignancies ( - Table 1 ). The primary indication of chemoport insertion was the requirement of long-term chemotherapy among the various cancer patients. Inclusion criteria included: 1) patient within the 13 to 75 years age group with a diagnosis of malignancy; 2) expected survival of 3 months or more with a treatment plan of IV chemotherapy.

All polyurethane chemoport catheters were put in the operating room by surgical oncologists on the opposite side of disease in breast cancer patients and right subclavian vein in other cancer patients either under general anesthesia at the time of primary surgery or under local anesthesia in other patients. A single chamber chemoport with a 9.6 $\mathrm{F}$ polyurethane catheter was used. We inserted the needle in the deltopectoral groove, punctured the subclavian vein blindly (or under sonographic guidance, if required), and put the guidewire into the vein. A dilator and peel-away sheath were threaded over the guidewire, and polyurethane catheter was placed using Seldinger's technique under the guidance of fluoroscopic "C" arm. A separate incision of size approximately $3 \mathrm{~cm}$ was made on the chest wall at the level of 2 nd rib. A subcutaneous pocket was made for chemoport chamber, and catheter brought out in this wound with the help of a tunneller. The length of the catheter was measured at $0.5 \mathrm{~cm}$ below the tip of a curved artery placed at the sternomanubrial joint and confirmed in the ' $\mathrm{C}$ ' arm. We attached the catheter with the chemoport and checked the backflow. Heparinized saline was used to flush the port and catheter. Huber's needle was put if the patient required chemotherapy in the same sitting.

In our institution, we routinely do a chest X-ray in each patient to diagnose early complications. We start chemotherapy on the same day, if required, after viewing the chest X-ray.

Patients were followed-up till removal of the chemoport due to any reason like completion of chemotherapy, complications requiring premature removal, or patient's will.

Table 1 Various malignancies

\begin{tabular}{|l|l|l|}
\hline & Malignancies & $\mathbf{n ( \% )}$ \\
\hline 1. & Breast & $141(83.92)$ \\
\hline 2. & Lung & $09(5.35)$ \\
\hline 3. & Gastrointestinal & $8(4.76)$ \\
\hline 4. & Leukemia & $6(3.57)$ \\
\hline $\mathbf{5 .}$ & Ovary & $4(2.38)$ \\
\hline & Total & 168 \\
\hline
\end{tabular}

The study was approved by the Institutional Ethics Committee.

\section{Results}

The median age was 41.6 (range 13-64) years. Out of 168 chemoports inserted, 30 (17.85\%) complications were registered ( - Table 2). Six (3.57\%) were early (within 4 weeks of placement of port) and $24(14.28 \%)$ late complications (after 4 weeks).

Our two patients had malposition of the tip of the catheter; one in the ipsilateral internal jugular vein ( - Fig. 1) and in one case tip was reversed in the opposite subclavian vein. Reexploration was done in the first case and the tip location was corrected. The patient who had the catheter tip in the opposite subclavian vein continued with chemotherapy but blocked her catheter after two cycles of chemotherapy, so her catheter was changed under local anesthesia.

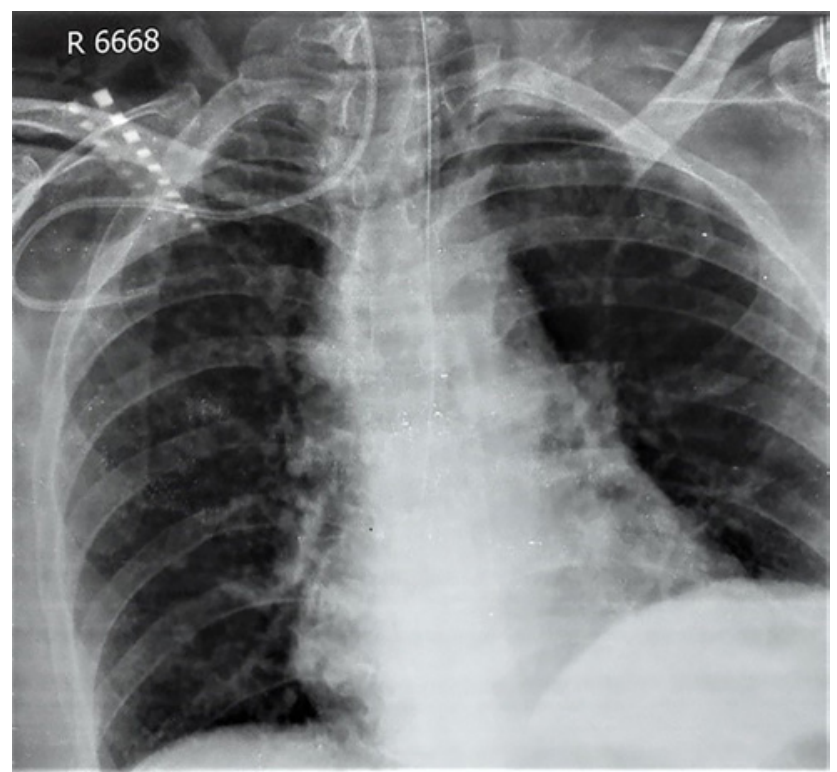

Fig. 1 Catheter in the right internal jugular vein.

Table 2 Complications

\begin{tabular}{|l|l|l|}
\hline & Complications & $n=168(\%)$ \\
\hline 1. & Malposition of catheter tip & $2(1.19)$ \\
\hline 2. & Pneumothorax & $1(0.59)$ \\
\hline 3. & Hematoma, seroma & $2(1.19)$ \\
\hline 4. & Deep vein thrombosis & $1(0.59)$ \\
\hline $\mathbf{5 .}$ & Fracture of catheter & $1(0.59)$ \\
\hline $\mathbf{6 .}$ & Reversal of port & $1(0.59)$ \\
\hline 7. & Excess length of catheter & $1(0.59)$ \\
\hline $\mathbf{8 .}$ & Infective complications & $16(9.52)$ \\
\hline $\mathbf{9 .}$ & Catheter kinking & $1(0.59)$ \\
\hline $\mathbf{1 0 .}$ & Catheter obstruction & $4(2.38)$ \\
\hline & Total & $30(17.85)$ \\
\hline
\end{tabular}


Hematoma and seroma occurred as early complications in two patients, and both were resolved after aspiration and pressure dressings.

Pneumothorax occurred in one patient diagnosed on postoperative chest X-ray ( - Fig. 2), which resolved spontaneously on conservative treatment only.

Patient of subclavian vein thrombosis presented with pain and swelling in the involved arm. She was diagnosed with venous Doppler study and her catheter was removed. The patient was advised low-molecular heparin.

Reversal of port is a rare complication. It can be diagnosed clinically by fingertips only and cannot be diagnosed by any imaging modality. It occurred in one patient and required opening of the wound, correction of the port, and resuturing of the wound under local anesthesia.

Fracture of the catheter (pinch-off syndrome) occurred in one patient after 110 days of chemoport placement. The catheter was found coiled in the right ventricle. The catheter was removed successfully by an interventional radiologist.

One patient had the tip of the catheter in the right ventricle diagnosed on postoperative chest X-ray ( - Fig. 3). Correction of catheter length was done in the same admission under local anesthesia.

Excessive angulation or kinking of the catheter is suspected by the absence of blood backflow and can be readily seen on X-ray (-Fig. 4). This may be due to either technical failure or characteristics of the catheter (thin-walled and malleable). It leads to nonfunctioning and premature removal of port.

Thrombosis of the catheter is a late complication, which occurred in five patients. In this, there is the formation of thrombus at the tip and a layer of fibrin on the external surface of the catheter. We tried to clear the thrombus with flushing and fibrinolytic agents but were unable to open the blockage in three patients, who required premature removal of the port

Infective complications occurred in $16(9.52 \%)$ patients. Out of 16 patients, 3 had port site infection and presented

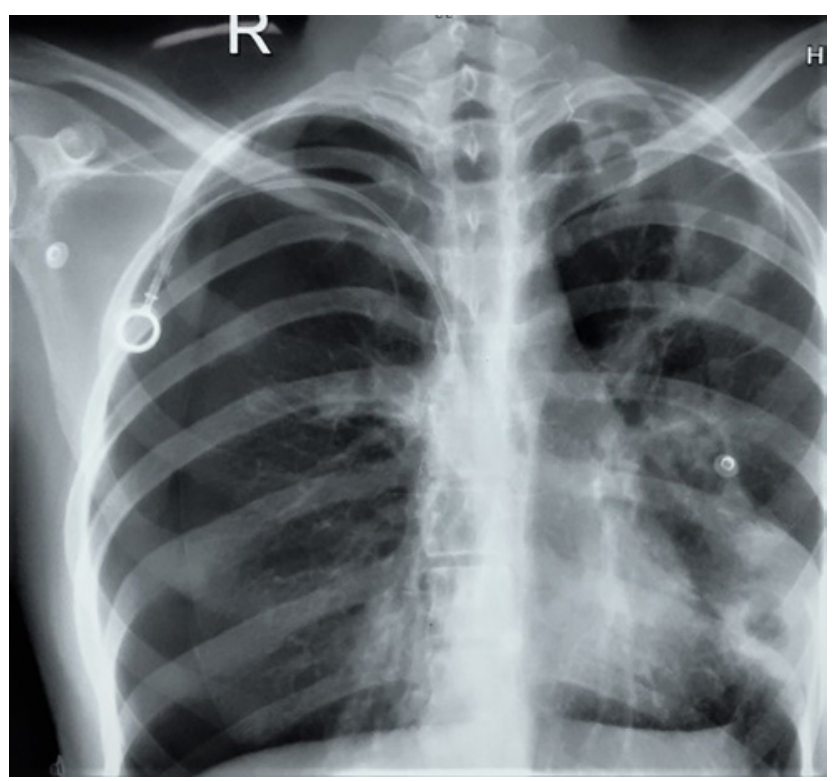

Fig. 2 Right pneumothorax. with pain and swelling. Chemoport was removed promptly in all 3 patients and antibiotics were administered as per the antibiogram. The other 13 had suspected catheter-related infection with a normal chemoport site. Paired blood culture for differential time to positivity (DTP) was sent. DTP was suggestive of catheter-related infection in 9 patients. Appropriate antibiotics were administered as per the antibiogram after the removal of the chemoport.

Out of 168 chemoports inserted, 17 (10.1\%) were removed prematurely due to mechanical or infective complications.

\section{Discussion}

In cancer patients, long-term access to the venous system for administration of chemotherapy is required as chemotherapy

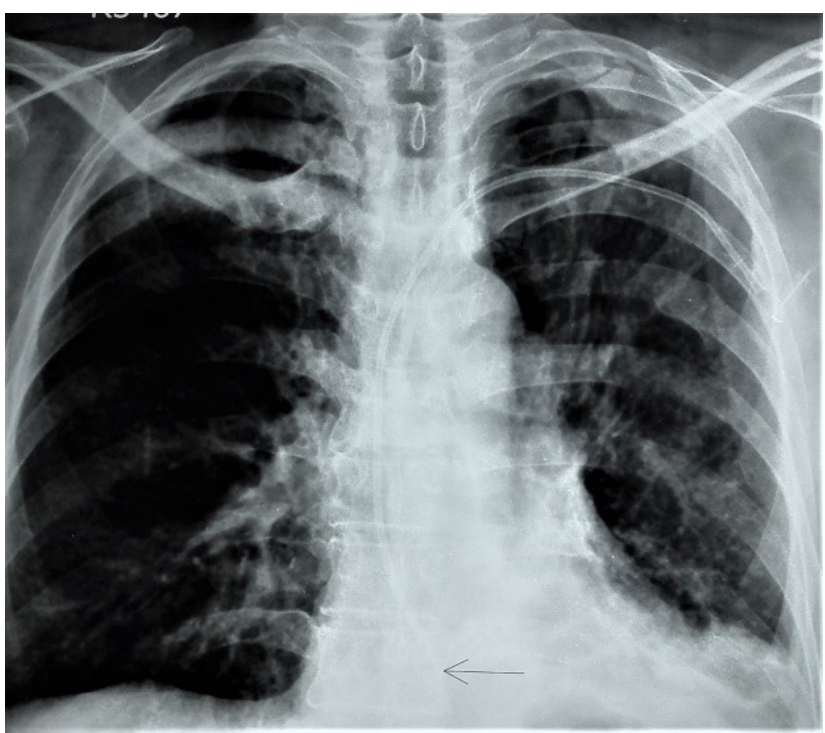

Fig. 3 Catheter tip in the right ventricle.

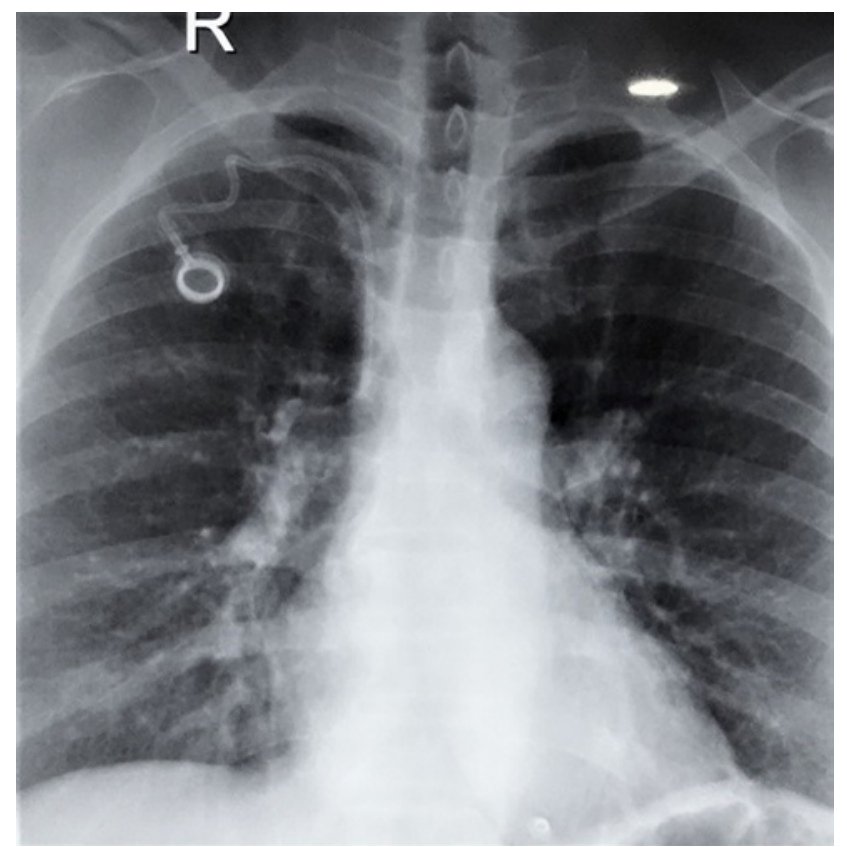

Fig. 4 Excessive angulation of the catheter. 
is administered in multiple cycles. Moreover, the vesicant nature of chemotherapeutic agents, frequent venous sampling, and the administration of parenteral supportive care at frequent intervals makes it necessary to have a long-term venous access system. After the advent of chemoport, all these can be achieved with minimal and acceptable complications and high-patient satisfaction. We conducted this study among various cancer patients like breast, lung, gastrointestinal, leukemia, and ovary cancer. We had the maximum number of breast cancer patients in our series. These patients require special consideration as after surgery with axillary lymph node dissection, venous access is avoided in the ipsilateral limb.

There are two types of catheter systems polyurethane (open) and Groshong's (valved), which are available with the same type of chemoport. Groshong's catheter has a valve at the tip. The opening of the valve is dependent on the application of either negative or positive pressure. In resting conditions, the valve remains closed and there is theoretically less chance of formation of thrombus at the catheter tip and hence fewer thrombotic complications. However, it has been demonstrated that the use of a Groshong's catheter is not superior to an open catheter system in terms of early and late complications related to the device and its use. ${ }^{4}$

Our success rate of blind cannulation of the subclavian vein was $97.02 \%$ (163 out of 168 patients), as ultrasound (US) guidance was required only in 5 (2.97\%) patients. Presently, US-guided puncture of the subclavian vein is a safe technique and standard of care as it has a low periprocedural complications rate. ${ }^{5}$

In our study, infective complications were higher (9.52\%) as compared with other studies (4.3\%). ${ }^{6}$ Chemoport handling should be done by a trained health care worker only. Appropriate and aseptic handling of chemoport does not lead to increased infective complications, even if the chemotherapy is administered at home. ${ }^{7}$ Prophylactic antibiotics have been used by some authors to reduce these complications. However, there is no clear evidence showing any decrease in the infection rates. ${ }^{8,9}$

Many patients prefer peripheral cannula for chemotherapy rather than chemoport due to financial reasons. The difficulty in finding the peripheral veins for cannulation is related to the number of chemotherapy cycles given to the patient. Chemoport placement by surgeons had been reported to be more cost-effective than when interventional radiologists perform this procedure. ${ }^{10}$

Chemoport should be removed after some time when treatment had completed. This may vary in different institutions. Ignatov et al reported that $74 \%$ of patients wanted to keep chemoport in situ for further use. ${ }^{11}$ Contrary to this, Kreis et al recommended prompt removal of chemoport after treatment, as it requires periodic flushing and adds to the overall cost. ${ }^{12}$

\section{Conclusion}

Complication rates are low with polyurethane catheter system. Placement of chemoport and its maintenance requires a dedicated team approach along with patient education and follow-up. Early identification of complications and proper management is necessary to avoid morbidity and mortality associated with its use.

\section{Funding}

Nil.

\section{Author's Contribution}

All authors contributed to the study conception and design. Data collection and analysis were performed by GSM, NNB, and DS. The manuscript was written by DS, AS, and GSM. Manuscript review and editing was done by AS and DS. All authors read and approved the final manuscript.

\section{Conflict of Interest}

The authors declare that they do not have any conflict of interests.

\section{References}

1 Niederhuber JE, Ensminger W, Gyves JW, Liepman M, Doan $\mathrm{K}$, Cozzi E. Totally implanted venous and arterial access system to replace external catheters in cancer treatment. Surgery 1982;92(4):706-712

2 Munck A, Malbezin S, Bloch J, et al. Follow-up of 452 totally implantable vascular devices in cystic fibrosis patients. Eur Respir J 2004;23(3):430-434

3 Davies MG, Feeley TM, Moore DJ, Shanik GD. Home parenteral nutrition using a totally implanted subcutaneous venous access device. Br J Clin Pract 1990;44(12):750

4 Biffi $\mathrm{R}$, De Braud F, Orsi F, et al. A randomized, prospective trial of central venous ports connected to standard open-ended or Groshong catheters in adult oncology patients. Cancer 2001;92(5):1204-1212

5 Biffi R, Orsi F, Pozzi S, et al. Best choice of central venous insertion site for the prevention of catheter-related complications in adult patients who need cancer therapy: a randomized trial. Ann Oncol 2009;20(5):935-940

6 Nishinari K, Wolosker N, Bernardi CV, Yazbek G. Totally implantable ports connected to valved catheters for chemotherapy: experience from 350 Groshong devices. J Vasc Access 2010;11:17-22

7 Brown DF, Muirhead MJ, Travis PM, Vire SR, Weller J, Hauer-Jensen M. Mode of chemotherapy does not affect complications with an implantable venous access device. Cancer 1997;80(5):966-972

8 Torramadé JR, Cienfuegos JA, Hernández JL, et al. The complications of central venous access systems: a study of 218 patients. Eur J Surg 1993;159(6-7):323-327

9 Brothers TE, Von Moll LK, Niederhuber JE, Roberts JA, Walker-Andrews S, Ensminger WD. Experience with subcutaneous infusion ports in three hundred patients. Surg Gynecol Obstet 1988;166(4):295-301

10 Sticca RP, Dewing BD, Harris JD. Outcomes of surgical and radiologic placed implantable central venous access ports. Am J Surg 2009;198(6):829-833

11 Ignatov A, Hoffman O, Smith B, et al. An 11-year retrospective study of totally implanted central venous access ports: complications and patient satisfaction. Eur J Surg Oncol 2009; 35(3):241-246

12 Kreis H, Loehberg CR, Lux MP, et al. Patients' attitudes to totally implantable venous access port systems for gynecological or breast malignancies. Eur J Surg Oncol 2007;33(1):39-43 